\title{
A novel microbond bundle pullout technique to evaluate the interfacial properties of fibre-reinforced plastic composites
}

\author{
PADMANABHAN KRISHNAN@ \\ Department of Manufacturing, School of Mechanical Engineering, VIT-University, Vellore 632014, India \\ padmanabhan.k@vit.ac.in
}

MS received 3 April 2016; accepted 14 October 2016; published online 26 July 2017

\begin{abstract}
The interfacial properties of the fibre composite systems decide the overall usability of a composite in simple and complex shapes, as they are the deciding factors in determination of the mechanical properties, structural properties and above all a complete understanding of the reliability of composite systems. In the present investigation, the interfacial properties of carbon fibre/epoxy composites viz., matrix shrinkage pressure, interfacial frictional stress, interfacial shear stress and coefficient of friction were evaluated through a novel microbond bundle pullout test. This test is different from the single fibre pull out, fibre fragmentation or the fibre push in test. Based on some of the physical principles involving the single fibre microbond pullout test, like the contact angle of the microbond matrix drop with the fibre surface, the surface tension/energy of the two surfaces before and after adhesion and the interfacial fibre/matrix chemistry, this is simple to perform and statistically averaged mesomechanical test is also easy to evaluate and is shown to be a test method that enables a conservative prediction of the laminate level or macromechanical shear properties of fibre composite systems. This test demonstrates the validity of the mesomechanical tests that are more relevant to the macromechanical tests than the micromechanical tests. Fractography carried out to corroborate the observed mechanical properties with the fracture features is also reported. The general advantages of the mesomechanical interfacial tests over those based on micromechanical assumptions is also discussed along with some common limitations.
\end{abstract}

Keywords. Microbond bundle pullout test; carbon/epoxy; fibre-reinforced composites; mesomechanics; interfacial properties.

\section{Introduction}

The interfacial properties of the composite systems decide the overall usability of a composite in simple and complex shapes, as they are the deciding factors in determination of the mechanical properties, structural integrity and above all a complete understanding of the composite systems. The production of carbon fibres and the studies on carbon fibre/matrix interfaces are well documented [1,2]. In the present investigation, the interfacial properties of a commercial industrial-grade carbon fibre/epoxy composite were evaluated through a new microbond bundle pullout test.

For a detailed reference on the earlier interfacial tests and methods that were designed, developed and conducted, the reader is directed to Kim and Mai's book [3]. In 1993, Sastry and co-workers [4] developed a test method, in which the microbundle pullout concept was tested through a single fibre pull out from a bundle encapsulated in an epoxy matrix. This test method was not statistically averaged like the current attempt and no clear correlation of the interfacial shear stress (IFSS) could be made with the adopted volume fraction. The present test was developed independently by the investigator and it is to an extent based on an earlier unpublished work [5]. It has been improved to an appreciable level through refinement in test parameters, as an earlier presentation was on a different system [6]. It is partly based on the physical principles involving the single fibre microbond pullout test [7]. It is an easy tool to measure the interfacial properties as it is easy to formulate, conduct and evaluate. It is a statistically averaged test, which is more realistic than the micromechanical single fibre pullout test. The properties obtained, however, are mesomechanical in nature and do not provide macroscale properties that can be obtained only in laminated products or components, as size and scale are involved.

\section{Experimental}

The microbond bundle test samples were prepared as follows:

A long bundle of the provided carbon fibres was used to deposit drops of the mixed resin and hardener on the surfaces through a wooden needle in gaps of about $50 \mathrm{~mm}$ along the length in tandem and left to cure when the drop sizes were uniform. This was achieved by gently spinning the bundle with both the hands to get uniform droplets of the resin. The cured droplets were examined under the scanning electron microscopy (SEM) to measure the droplet dimensions and the wetting contact angle. This is illustrated in figure 1a and $b$. The cured fibre bundle samples were cut to $25 \mathrm{~mm}$ in gauge length with each gauge length bonded with one 


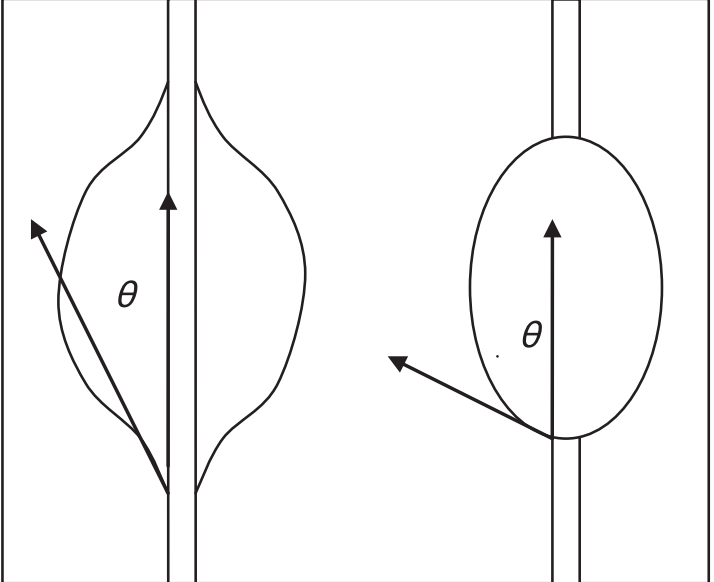

(a)

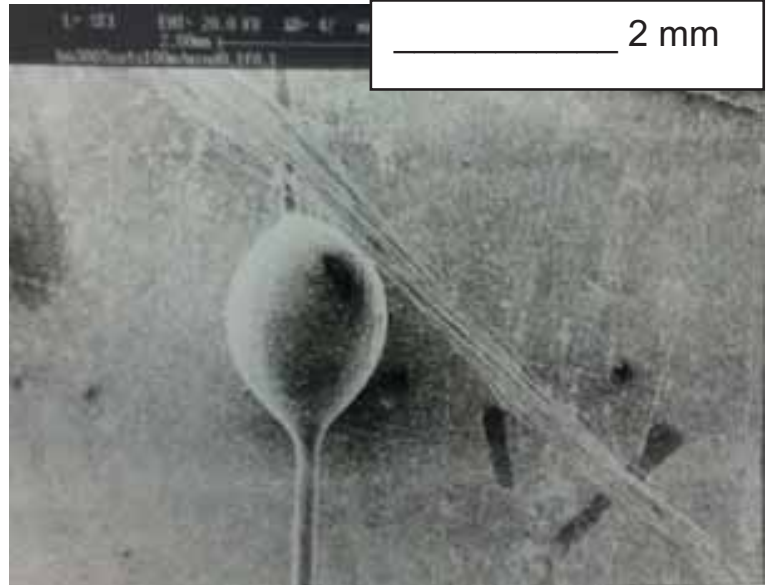

(b)

Figure 1. (a) Schematic of the angles of the cured droplets and (b) SEM image of a cured epoxy droplet.

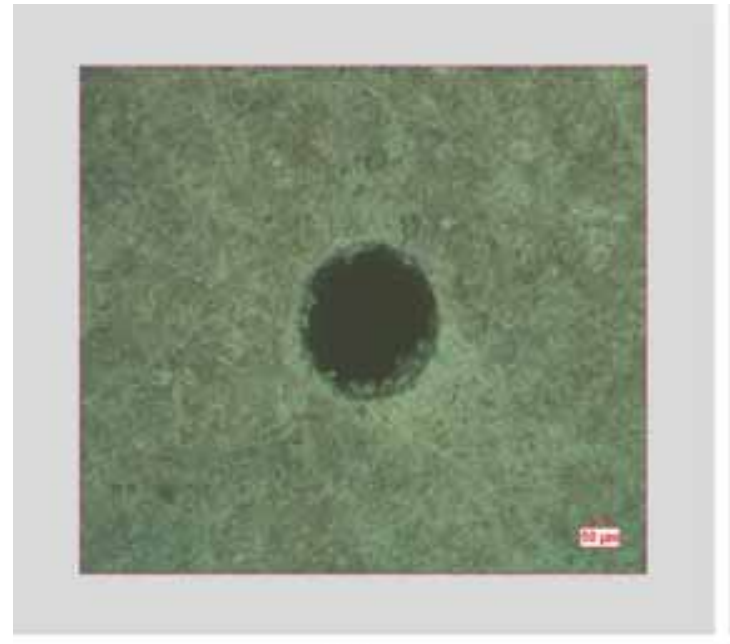

(a)

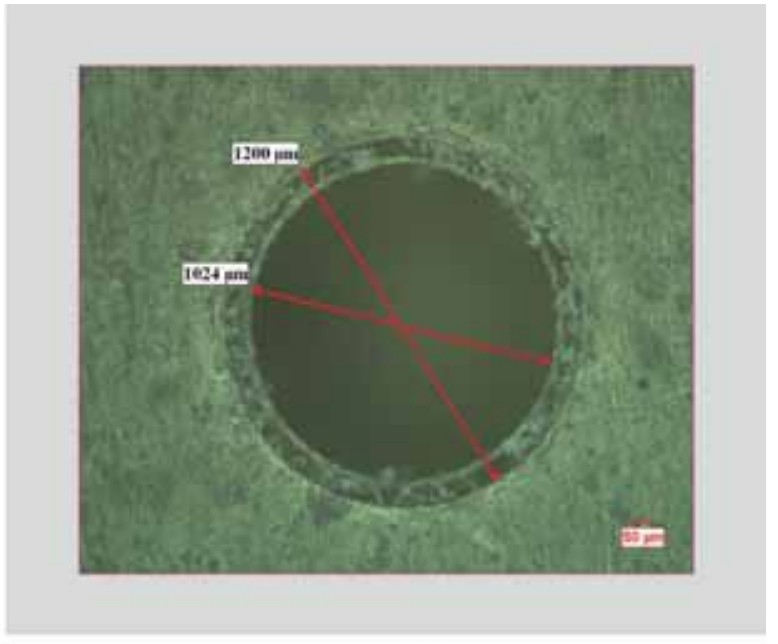

(b)

Figure 2. (a, b) Optical micrographs of electrical discharge machining drilled microvises with approximate diameters of 0.5 and $1 \mathrm{~mm}$.

droplet and attached to card board tabs at the opposite end of a microvise held to the tensile grips as shown in figures 2 and 3. These samples were cut with a tuft of the fibre seen out at the resin droplet end to enable frictional shearing/sliding of the droplet through the microvise after debonding during testing.

The samples were tested by Instron 8801 UTM at a pulling rate adopted for tensile testing procedure for composites $\left(5 \mathrm{~mm} \mathrm{~min}{ }^{-1}\right)$ through a microvise plate, illustrated in figures 2 and 3. The microvise was firmly held by screws in an aluminium cage tightly gripped in the UTM with load cells, whose maximum capacity was $100 \mathrm{~N}$ or $1 \mathrm{kN}$. It was taken care that the maximum load experienced by the specimen was only about $20 \%$ of the overall capacity of the load cell in order to avoid compliance-related problems. The samples that failed in the desired shear pullout mode were selected for gold coating prior to SEM observation. The observed fracture features are discussed in the section on fractography. The other specimens that failed in the undesirable tensile fibre fracture modes were also examined for comparison and understanding the embedded length limits of the resin for a fibre tensile fracture.

\section{Theoretical considerations}

\subsection{Micromechanical approach}

The interfacial parameters were evaluated from the requisite micromechanical parameters using the available data shown 


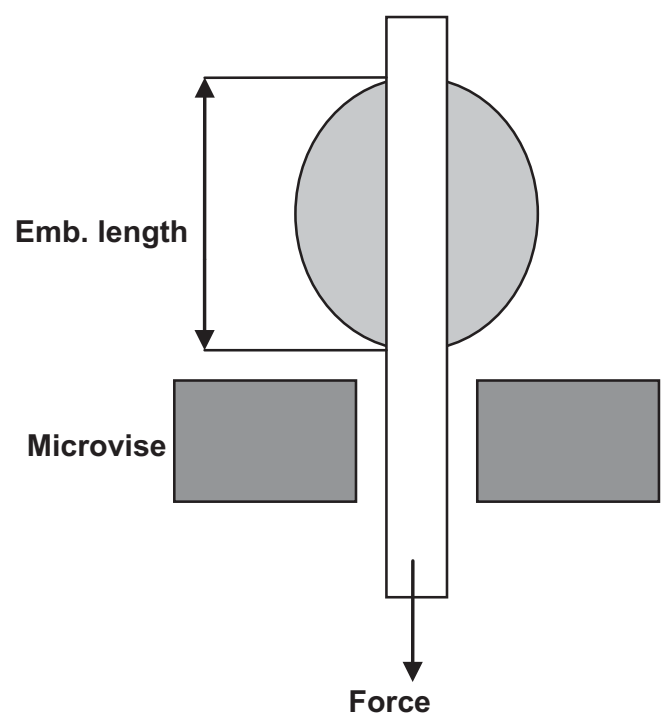

Figure 3. The microbond fibre bundle pullout test and the involved parameters.

Table 1. Features of the test specimens and methods.

Type of fibre: carbon fibre industrial grade (tensile strength $=2.36$ $\mathrm{GPa}$ ) and modulus $E_{\mathrm{f}}=120 \mathrm{GPa}$

No. of filaments in a bundle $=600$

Resin: high-performance standard araldite epoxy adhesive

Elastic modulus of cured resin, $E_{\mathrm{m}}=2.2 \mathrm{GPa}$

$V_{\mathrm{f}}$ in the bundle section $=0.14$

Average bundle $\Phi$ in composite $=0.428 \mathrm{~mm}$

Radius of bundle, $r=0.214 \mathrm{~mm}$

Microvise $\Phi=465 \pm 5 \mu \mathrm{m}$ and $1 \mathrm{~mm}$

Average diameter and length of the resin drop, variable

in table 1 for the concerned materials and testing conditions and a computation software called CADEC [8]. Though these results cannot be expected to be in good agreement with the present tests that fall in the mesomechanical domain, the same was carried out due to its established relationships and proximity to the mesomechanical approach as it would enable to study and understand not only the differences between the two approaches but also the need to develop mesomechanical formulations further on a brick by brick basis.

\subsection{Microbond bundle pullout test}

As discussed in the previous section and below, there seems to be a need to develop a microbond bundle pullout test that is comparable in all aspects to the semi-empirical resin slab/fibre bundle pullout approach [9]. The improvement of the known draw backs of the single fibre microbond pullout method [7], such as the difficulty in formulation and testing (with lack of provision for volume fraction of fibres in the cylindrical assemblage) and the multiple bundle pullout method which is laborious to evaluate but easy to conduct, are discussed here. The microbond bundle pullout performance of the test described here was conceived and evaluated for the first time and independently. The underlying and governing principles are similar to that of the microbond single fibre pullout test method, but the rationale followed in this investigation is clearly spelt out for reasons described.

The mean and the standard deviation values for the droplets and force plots are given in table 2 .

The evaluated average intrinsic bond strength, $\tau=13.52$ $\mathrm{MPa}$ at $V_{\mathrm{f}}=0.14$ and the evaluated average IFSS at failure $=56.23 \mathrm{MPa}$.

The perceived and the observed salient features of this test method are that it is easy to formulate and evaluate compared to any known interfacial test method. It requires no tooling or shaping. It is less cumbersome to perform than the single fibre microbond test [7] or the resin slab/bundle pullout test [9]. It has more physical relevance as it is mesomechanical in nature, well averaged in results and more precise than the microbond single fibre pullout test. It addresses the issue of volume fraction similar to the bundle pullout test, which the single fibre pullout tests cannot give. The cylindrical assemblage of fibres in the bundle region, which is called the CAM model of fibres, is considered here. It is limited only by a consistent drop size and wetting angle and may not be applicable to thicker fibre bundles that are used in high GSM (grams per square metre) fabric materials. However, pressure is never considered in any of the fibre-matrix interfacial tests, but most of the laminates are fabricated with about 1-20 bars of pressure either with a vacuum bag or a compression moulding machine. The drop or the resin slab cure in the atmospheric conditions and the influence of external pressure on the interfacial shear properties of laminated composites is never manifested in all the microbond pullout tests. Hence, the estimates of the present technique are conservative as the positive influence of compacting pressure on the interfacial properties is never considered. The underlying equations for this method are mentioned here. The interfacial intrinsic bond strength, $\tau$, is calculated from [7]

$$
\tau=F / \pi \Phi l,
$$

where $F$ is the peak debonding force in newtons minus the initial frictional force, if any. In the present case, the microvise was optimally designed not to produce any frictional trace with the fibres or the adhering resin. $\Phi$ is the average diameter of the fibre bundle in $\mathrm{mm}$ and $l$ is the embedded length of the resin droplet in the fibre bundle in $\mathrm{mm}$. The interfacial intrinsic bond strength is given by the peak debonding force divided by the available outer interfacial area, provided the volume fraction is sizeable and failure is at the outer interface due to the stress distribution from the fibres to the matrix and if the matrix is considerably weaker than the fibre. This can also be verified by observance of fracture and failure. The same cannot be expected in smaller volume fraction samples with a larger bundle diameter, which is sometimes due to low matrix curing pressure or adhesion. In such cases the fibre distribution and larger interfacial area would lower the 
Table 2. Microbond bundle pullout test.

\begin{tabular}{lccc}
\hline Specimen ID & $\begin{array}{l}\text { Drop length } \\
(1), \text { mm }\end{array}$ & $\begin{array}{l}\text { Contact angle } \\
(\theta, \text { degrees })\end{array}$ & $\begin{array}{l}\text { Maximum force for } \\
\text { fibre pullout }(\mathrm{N})\end{array}$ \\
\hline 1 & 2.25 & 24.25 & 22.97 \\
2 & 4.42 & 12.5 & 64.85 \\
3 & 3.17 & 30.5 & 75.65 \\
4 & 2.97 & 20.5 & 81.25 \\
5 & 2.9 & 20.25 & 59.85 \\
6 & 2.84 & 22 & 32.52 \\
7 & 3.5 & 30 & 58.04 \\
8 & 4 & 22 & 70.00 \\
9 & 3 & 20 & 40.00 \\
10 & 4 & 15 & 75.01 \\
Mean & 3.09 & 21.7 & 56.18 \\
SD & 0.701 & 5.67 & 19.92 \\
\hline
\end{tabular}

intrinsic bond strength and the interfacial shear strength. Here, the interfacial area is governed by the sum of the cylindrical interfacial areas. However, the interfacial shear strength is still governed by the statistical average of the distance between the fibre cross-sections that lead to a matrix controlled or interfacial controlled fracture behaviour assuming that the interfacial conditions are prior knowledge.

A drop profile is illustrated in figure 1a and $b$. The wetting contact angle produces a force that compresses the resin against the fibre end acting against the shearing process. This force, $F_{\mathrm{c}}$, should be equal to $F \cot \theta, F$ being the simultaneous direct force that produces the compressive component. This can be debonding/adhesive, tack or frictional at any tentative location in the load deflection plot that has be understood with care and the component should not be considered as due to only the maximum force as previously given [7]. It manifests on frictional onset as a second component. Thus we can write

$$
F=F_{\text {ad }}+\mu F_{\mathrm{c}},
$$

where,

$$
F_{\text {ad }}=F_{\text {max }}=\tau A .
$$

$A$ being the considered interfacial cylindrical area. In this case, the maximum force is also the force required for debonding as indicated in table 2. However, the peak pullout force can occur after debonding due to build up of frictional stress which can again be addressed by equation 2 .

$$
F=\tau A+\mu F_{\mathrm{f}} \cot \theta
$$

Here $F_{\mathrm{f}}$ is a variable force down the load deflection plot unlike the peak debonding force that is singular, $\theta$ is the angle of wetting and contact of the fibre bundle with the resin droplet and $\mu$ is the static coefficient of friction as the pull out occurs at a rate of $1-5 \mathrm{~mm} \mathrm{~min}^{-1}$. It is evident from the load deflection plot (see figure 4) that the value of the coefficient of friction is likely to change from the onset of frictional sliding to a steady value, if the frictional sliding persists. The various stages of the failure process are already discussed in the schematic sketch shown in figure 4. IFSS is generated by dividing equation (4) with $A$ that also gives us the frictional stress values. Hence,

$$
F / A=\operatorname{IFSS}=\tau+\mu \tau_{\mathrm{f}} \cot \theta
$$

We take a statistical view to failures as shown in figure 5, where the length of the drop is shown to vary from about 1.5$4 \mathrm{~mm}$. It is difficult to form a droplet beyond $4 \mathrm{~mm}$ length for a bundle of this size and fibre breakage is also appreciable due to the critical length being exceeded that favours a tensile failure. So within the working window, one may get as many data points as possible for a desired shear pullout failure without damaging the fibres in the sheared area.

\section{Results and discussion}

The pullout force $v s$. drop length results from the pullout tests are plotted in figure 5. The average slope of the force $v s$. embedded length for the microbond bundle pullout tests produces an $x$-axis intercept of $0.35 \mathrm{~mm}$, indicating that the pullout force is zero when the drop size is zero. The experimental offset of $0.35 \mathrm{~mm}$ can be considered as deviation from this physical truth - a small error in averaging. The variance is also appreciable in this test as the cylindrical area of contact of the resin with the fibre bundle varies with diameter of the bundle and the embedded drop length. This causes the pullout force to increase with increasing embedded drop length assuming that the fibre bundle has constant diameter. The IFSS results from the micromechanical formulations are 


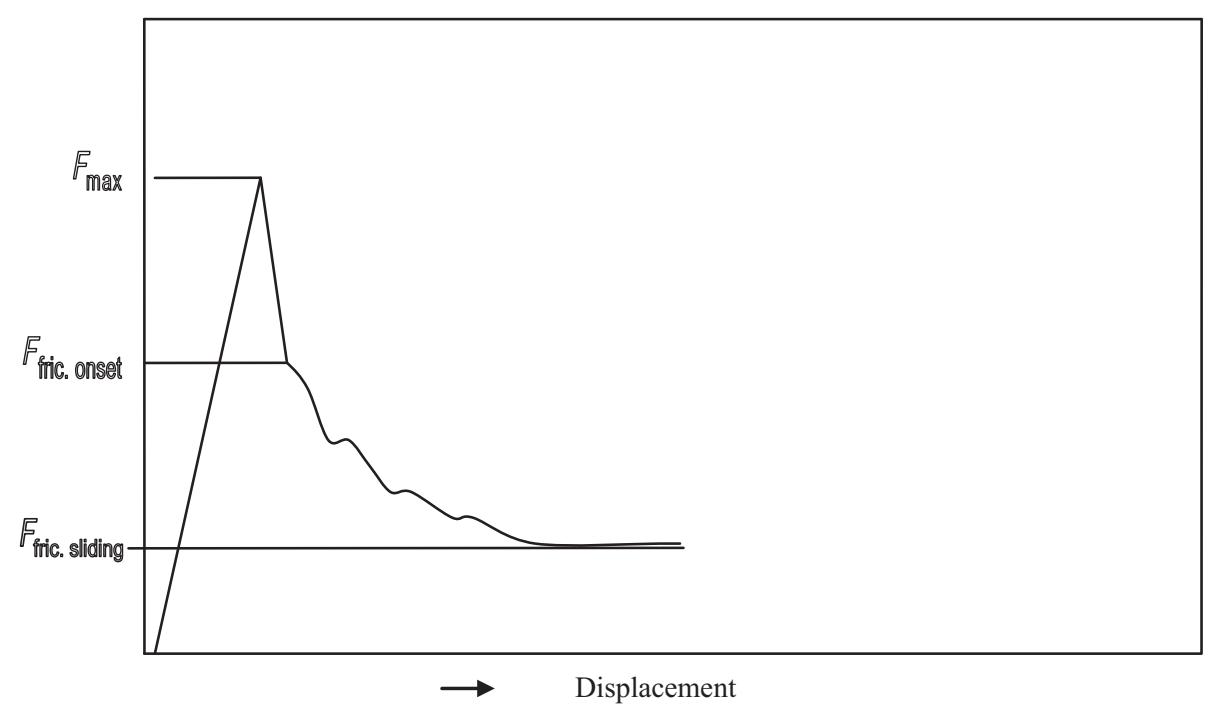

Figure 4. A schematic pullout force $v s$. displacement plot for a multiple fibre microbond pullout test.

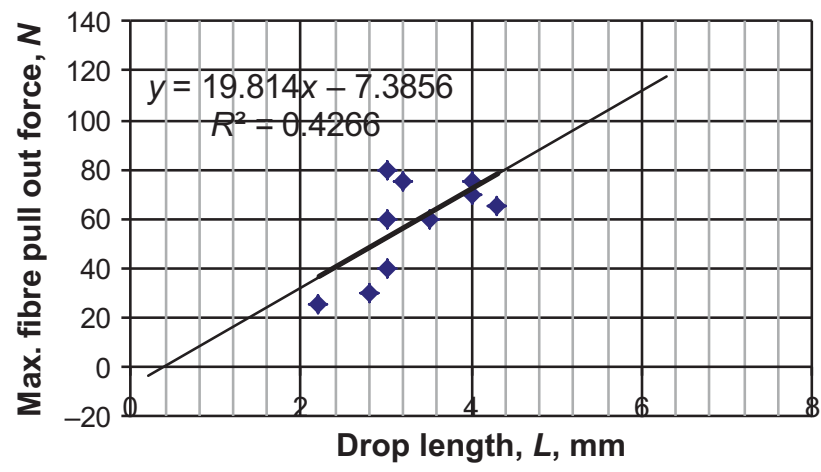

Figure 5. Maximum pullout force $v s$. drop length.

illustrated in figure 6 and compared with the laminate level and microbond test results.

The interfacial shear strength obtained according to Rosen's formula [8] is compared with that obtained with the Chamis formula [8] for any volume fraction of the fibre in the CAM region. These data are again compared, as shown in figure 6 , with the microbond data obtained through our experiments and the laminate interlaminar shear strength (ILSS) data for a similar carbon/epoxy composite at a specified volume fraction. It is seen that there is a distinct difference between the conservative IFSS values obtained through the micromechanical route and the mesomechanical values obtained in this experimentation. The mesomechanical evaluation is seen to be closer to the real and true macromechanical values obtained elsewhere.

Using this relationship the IFSS of the composite can be found. It is seen that the wetting contact angle of the fibres with the matrix droplet is determined by the shape that the droplet assumes. Some droplets are formed with a toe that has a short but linear portion near the ends that determines the contact angle, while quite a few others were cleaner arcs that did not have a toe region and direct in the contact angle measurement. Some of the drops did not have any consistent linear toe making it impossible to measure the lower of the two exhibited contact angles (see figure 1).

Hence, it is seen that the evaluation of IFSS produced an upper bound and a lower bound for the same test method due to the variations in the contact angle in the range of $12.5^{\circ}-30.5^{\circ}$. A lower and upper bound of $22.97-81.25 \mathrm{~N}$ of pullout force is achieved in the observed contact angle measurement ranges. For all practical purposes, the average IFSS value of 56.23 $\mathrm{MPa}$ can be considered to be reliable and within the range.

The observed and the evaluated data are given in tables 1 and 2 and compared with the theoretical predictions based on micromechanical approach, as shown in figure 6. It is seen that the mesomechanical semi-empirical evaluation is equal to or higher than the micromechanical predictions of Rosen on Chamis. Normally, it is seen that the values of IFSS obtained from the microbond test for a single fibre pullout exhibit the lowest values among all the known methods that measure IFSS. The present technique proves that the obtained values are not a testing artifact but an attribute to the mesomechanical and statistical nature of these tests. The ILSS at any of these volume fractions is expected to be higher if not equal to the experimentally evaluated IFSS values, as laminates are normally compacted at higher pressures, with a few defects as dislocation sites (to improve the ILSS up to a certain percentage of defects). Besides, the ILSS is an indirect method to test the interfacial properties as against the IFSS pullout tests that are direct. This seems to hold even for ILSS data evaluated for the carbon fibre composites elsewhere [2,3]. This is a significant finding of this investigation that adds a reliable approach to the evaluation of interfacial properties through 


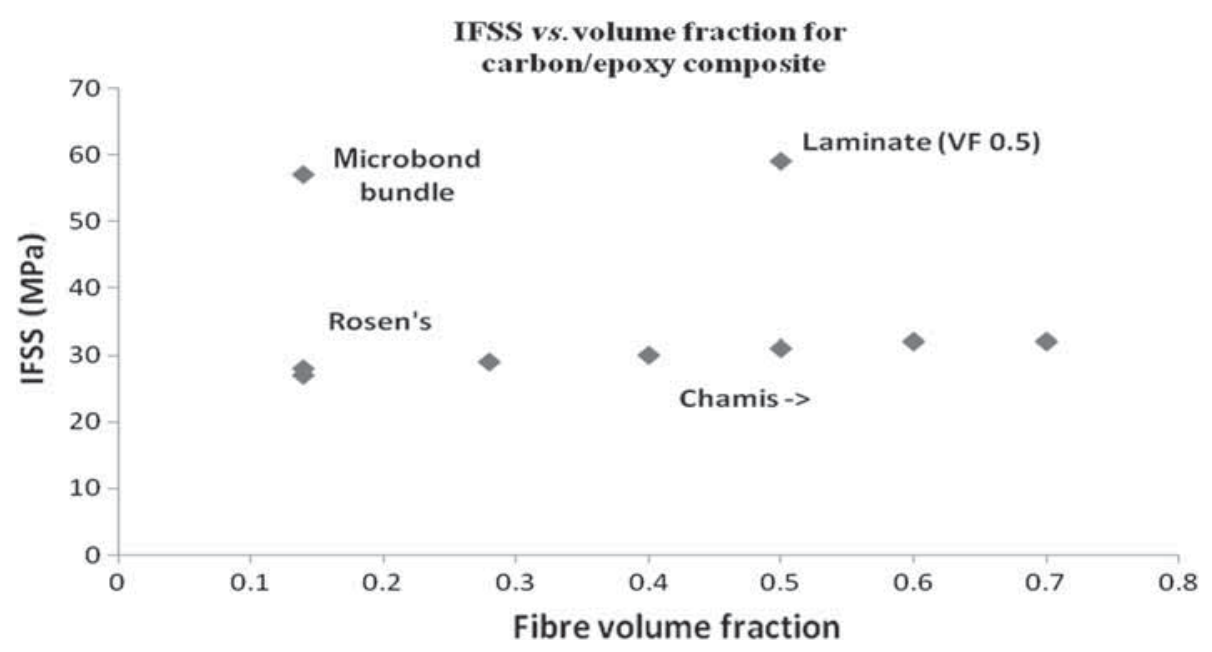

Figure 6. IFSS vs. volume fraction of fibres.

the mesomechanical route as fibre bundles and the ILSS test are mesoscale in nature.

The values obtained from the useful microbond bundle pullout test can thus be used to evaluate and compare the test data obtained from the multiple bundle pullout test conducted earlier and reported elsewhere [9]. It must be noted that the frictional sliding aspects are more addressed in the microbond bundle test due to the longer and consistent shearing/sliding of the drop against the bundle and to a lesser extent in the multiple fibre pullout test due to the embedded resin fracturing and disintegrating providing no avenue for the frictional sliding that a drop undergoes. The size and shape of the drop or the resin slab dictate the fracture modes and the upper critical length required for a tensile fracture of the fibre bundle. At a given slab thickness in a multiple fibre pullout test [9] or the drop length in a multiple microbond test that provides a valid shear pull out, the resin slab volume and dimensions are more and the disintegration possibilities are more. Hence, the load deflection plots of microbond fibre bundle tests exhibit more shear and frictional sliding, while the resin slab fracture is more catastrophic. The static coefficient of friction, $\mu$, at the onset and frictional sliding can be evaluated from a knowledge of the direct tensile force and the corresponding lateral compressive force, $F_{\mathrm{c}}=F \cot \theta$. Since $\mu$ is the lateral force divided by the normal (direct) force in this case, it can be evaluated for conditions of onset and sliding. However, as per this definition the coefficient of friction is a two-dimensional quantity and since the contact angle can be measured on either side of the fibre bundle, the force $F / 2$ $\cot \theta$ is obtained by dividing the corresponding $\operatorname{direct} F$ for a precise estimation of $\mu$ at onset and then sliding. In the present case, the two phenomena are fairly consistent and deducible. An evaluated value of $\mu_{\text {onset }}$ is 1.256 and $\mu_{\text {sliding }}$ is variable but lesser. The matrix shrinkage pressure is approximately the frictional interfacial stress divided by the static coefficient of friction at onset. So the matrix curing shrinkage pressure is about $44 \mathrm{MPa}$ in a resin drop at onset as it is the frictional interfacial stress divided by the static coefficient of friction, though fibre surface and resin geometry are certain to play a role in the evaluation of the matrix curing shrinkage pressure over a fibre surface area.

For the same volume fractions one may have different bundle diameters or for the same bundle diameter one may have different volume fractions, thereby causing a change in the cylindrical area of the bundle and its contact with the cured resin around it. If the volume fraction of the fibres is too less, like 0.1 or 0.05 and the matrix shrinkage pressure is less, the fibres would tend to form a bigger diameter and the contact area of the cylindrical assemblage with the surrounding resin would be more. If the matrix shrinkage pressure is more the cylindrical area would be lesser as fibres tend to cluster and the contact area with the surrounding resin would be lesser. Hence, for a low volume fraction of the fibres and a low matrix curing shrinkage pressure, the cylindrical assemblage of the fibres would have fewer fibres at the walls of the cylinder and the shear strength evaluation will be inaccurate. Volume fraction can be chosen with a knowledge of the dTex (number of fibres) of a fibre bundle, given the diameter of a single fibre, and the matrix curing shrinkage pressure, if it is a thermoset, and the solidification shrinkage pressure, if it is a thermoplastic. But there has to be a trial and error approach to understand the relationship between the number of fibres with a diameter, the bundle diameter with fibre to fibre contact (as in a hexagonal array) and the matrix shrinkage pressure. In the present case as shown in table 1 , the $V_{\mathrm{f}}$ is $0.14-0.2$ and the matrix shrinkage pressure is $44 \mathrm{MPa}$, which is good enough to shrink the fibre bundle to a smaller and closer radius (as evinced later from fractography). As only the surface area of the fibre bundles sees interfacial pullout completely, the same is considered for the evaluation. The results are an average of the surface fibres and not the interior fibres that have their matrix either intact or cracked. The matrix shrinkage 


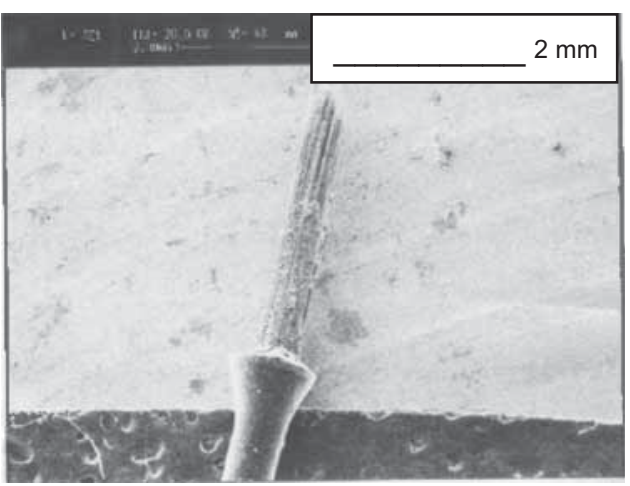

(a)

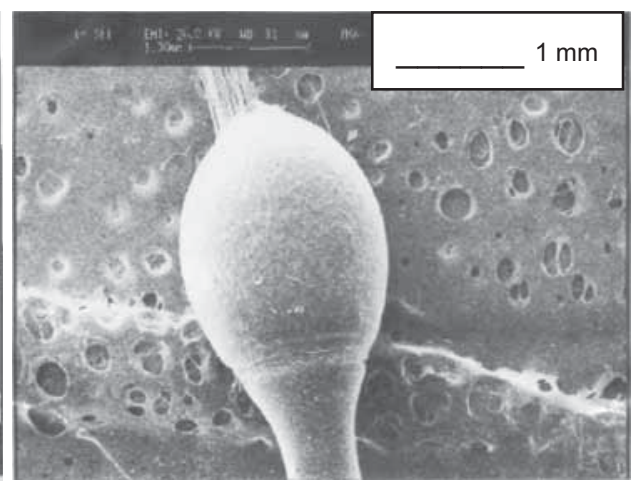

(b)

Figure 7. (a) The fibre bundle intact after the shear pull out of the bond. (b) SEM picture of the resin microbond droplet sheared out of the fibre bundle. Notice that the drop is still intact in this case.

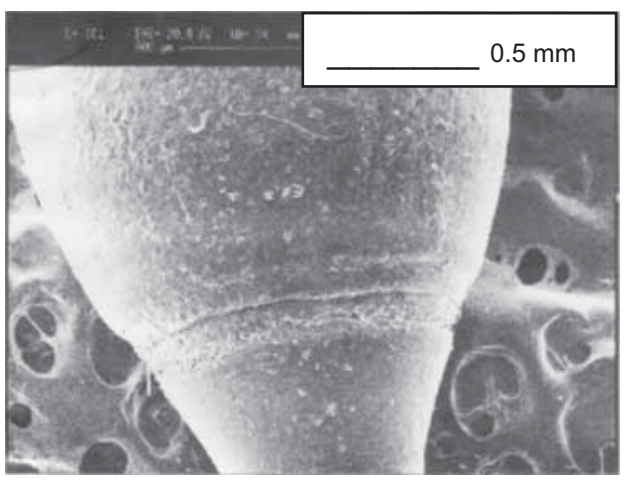

(a)

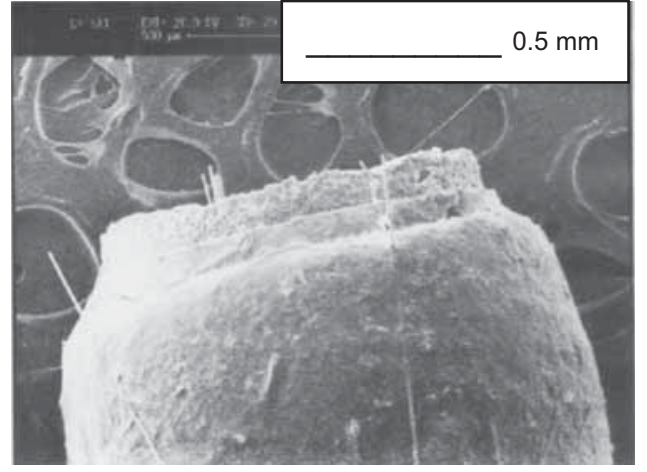

(b)

Figure 8. (a) SEM fractograph of the microbond sheared through the fibre bundle. Notice the microvise mark on the resin droplet due to compressive stresses near the exit. (b) A completely sheared out intact drop from the bundle of fibres due to pull out.

pressure manifests as coefficient of friction and the frictional stress developed at the onset of pullout sliding. The frictional stress at onset is equal to the coefficient of friction multiplied by the matrix shrinkage pressure. Therefore,

$$
\tau_{\mathrm{f}}=\mu\left(P_{\mathrm{o}}+P_{\mathrm{a}}\right)=1 / 2 \pi r\left(\mathrm{~d} F_{\mathrm{d}} / \mathrm{d}_{\mathrm{L}}\right),
$$

where $P_{\mathrm{o}}$ is the matrix curing shrinkage pressure and $P_{\mathrm{a}}$ is the poisson contraction pressure or the stress relaxation pressure. Both these parameters contribute to the contact pressure, $P$, in pull out. The radius of the bundle as evinced from image analysis is $r . F_{\mathrm{d}}$ is the pullout force of the fibre bundle and $\left(\mathrm{d} F_{\mathrm{d}} / \mathrm{d}_{\mathrm{L}}\right)$ is the slope of the plot of maximum load $\left(F_{\mathrm{d}}\right) v s$. the embedded length $(L)$ for various samples, at the region where $L$ is greater than the critical length $\left(L_{\mathrm{c}}\right)$ of the fibre bundle. If the length were less than the critical length, the failure would not be valid.

It should be noted that the precision of any interface test on fibre composite systems does not centre around accuracy, but is only averaged with a margin of scatter that may vary from test to test. The author intends to take up an energy-based approach as a sequel to the present strength-based approach on microbond bundle pullout tests.

\section{Fractographic analyses}

Fractographic investigations carried out with the aid of SEM substantiate the results and provide evidences that support the results. The fracture features are shown in figures 7 and 8.

The fracture features exhibited by the pulled out specimens are shown in figures 7 and 8 . The compressive stresses experienced near the exit of the microvise, increase the IFSSs, which is purely due to the test geometry that is typical of any microvise-based pullout test. Typical failures, such as drop shear, fibre broom features, compressive fracture with microvise marks and complete shear pull out of the drop were noticed. Few of the drops also failed completely before total shear out and were not intact after the test. Padmanabhan [10] illustrates some of the attempts to evaluate the mesomechanical properties of fibre/polymer composites through the 
micromechanical route. For a curtain raiser on the topic of mesomechanics of composites, the reader is referred to a pioneering article by Piggott [11]. The failure behaviour observed in the present case establish the mesomechanical nature of the construction and the test method. At present, efforts are on to develop test methods that evaluate the interfacial properties of self-reinforced composites, which is a challenge as the fibre and the matrix are of the same material.

\section{Conclusions}

The following conclusions emerge from the study:

1. The interfacial properties of the carbon fibre/epoxy composite, such as the interfacial frictional stress, $\tau_{\mathrm{f}}$, the static coefficient of friction, $\mu$, the instantaneous IFSS, the intrinsic bond strength, $\tau$, and the maximum interfacial shear strength, $\tau_{\mathrm{i}}$, were predicted and evaluated with the aid of micromechanical theories and the newly developed microbond bundle pullout technique.

2. The methods involved provide us with the interfacial properties evaluated that are consistent within the experimental allowables. The average interfacial shear strength is $56.23 \mathrm{MPa}$ from the bundle microbond pullout test performed at $V_{\mathrm{f}}$ of 0.14 . Higher volume fraction predictions are also made as practical impossibilities of achieving higher volume fractions of fibre bundles that exist in the microbond tests due to the lack of pressure in forming the resin drops.

3. An important finding of this investigation is that the mesomechanical evaluation of the interfacial properties is more reliable than the micromechanical predictions or their single fibre experimental evaluations due to their comparable values with the laminate level properties, raising new hopes on the precision (but not accuracy) of the strength-based small specimen testing approaches.

4. There is a mesomechanical domain that appears to be different from the micro or the macromechanical domain. This domain is significant as most of the macromechanical properties depend on mesomechanical fracture features, such as craze, crack tip plasticity, fibre bundle debonding from matrix and damage tolerance, to mention a few.
5. The fractographic investigations substantiate the results obtained, thereby throwing more light on the associated fracture mechanisms and the tools to be adopted to improve the techniques after understanding the fracture and failure modes.

\section{Acknowledgements}

The author thanks the CAMPT-VIT, Vellore, a FIST-DST facility for the Instron 8801 testing of the carbon/epoxy specimens. He also thanks M Ramya for the assistance. A part of this paper was presented in the INCCOM-13 conference at VSSC Trivandrum in 2014 and the author thanks them for the comments. The Aeronautics Research and Development Board, New Delhi, are thanked for the funded Project Number $1650 / 2012$.

\section{References}

[1] Shindo A, Fuji R and Sengoku M 1962 Japanese Bureau of Industrial Technics, Japanese Patent 4405/(13.6.62)

[2] Hughes J D H 1991 Compos. Sci. Technol. 4113

[3] Kim J K and Mai Y W 1998 Engineered interfaces in fibre reinforced composites (Amsterdam: Elsevier)

[4] Sastry A M, Phoenix S L and Schwartz P 1993 Compos. Sci. Technol. 48237

[5] Padmanabhan K 2002 Interfacial properties of Zylon AS/epoxy composites, Toyobo Confidentiality Agreement Report (Toyobo Co. Ltd, Japan) May 2002

[6] Padmanabhan K and Yue C Y 2002 Interfacial properties of Zylon AS/epoxy composites by the multiple fibre pull out technique - a case study. In: Proc-ACUN-4, UNSW, Sydney, Australia July 21-25, 238

[7] Miller B, Gaur U and Hirt D E 1991 Compos. Sci. Tech. 42 207

[8] Barbero E J 1999 Chapter 4. In: Introduction to composite materials design, CADEC (PA: Taylor and Francis)

[9] Yue C Y and Padmanabhan K 1999 Compos. B 30205

[10] Padmanabhan K 2003 The Singapore-MIT Alliance Report, Macrostructural property evaluation of fibre/polymer composites from the micromechanical behaviour through the mesomechanical route-Part 1, Innovation in Manufacturing Systems and Technology, NTU, Singapore, November 2003

[11] Piggott M R 1995 Compos. Sci. Technol. 5121 